\title{
Molecular characteristics of odorant-binding protein 1 in Anopheles maculipennis
}

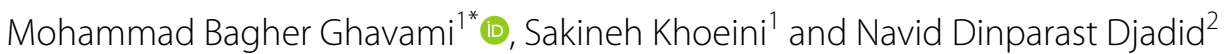

\begin{abstract}
Background: Anopheles maculipennis complex, the historic vector of malaria, causes serious medical problems worldwide and exhibits different behaviours. Studying the odorant-binding proteins (OBPs), which influence the chemosensory system and behavioural responses, is essential to understanding the population structure and developing effective control measures against this vector. The present study was designed to identify and analyse the obp 1 gene in An. maculipennis.

Methods: Adults of An. maculipennis sensu stricto were collected in Zanjan Province, northwest of Iran, and gDNAs of female mosquitoes were extracted. Fragments of An. maculipennis obp 1 (Amacobp1) gene were amplified using degenerate and specific primers, and some of amplicons were selected for sequencing.

Results: Analysis of amplified products identified that the sequence of Amacobp 1 gene was $1341 \mathrm{bp}$ long. This gene contains three exons ( $5^{\prime}$, internal, and $3^{\prime}$ of 160, 256, and 18 bp, respectively) and encodes 144 amino acids. The sizes of introns I and II in deduced gene are 268 and 358 nucleotides, respectively. The amino acid sequence in the C-terminal of AmacOBP1 is similar to that of major malaria vector Anopheles species. However, its $\mathrm{N}$-terminal has a specific signal peptide with 19 amino acids. This peptide is conserved in different studied populations, and its sequence of amino acids shows the most variation among anopheline species.

Conclusions: Degenerate primers in this study are suggested for studying obp 1 gene in Anopheles species. Amacobp 1 gene is proposed as a molecular marker for the detection of intraspecific ecotypes and diagnosis of different species within Maculipennis Group. Moreover, the N-terminal of AmacOBP1 peptide is recommended as a molecular marker to identify the Amacobp1 expression patterns in different chemosensory organs for assessing the molecular mechanisms and developing novel behavioural disturbance agents to control An. maculipennis.
\end{abstract}

Keywords: Anopheles maculipennis, Odorant-binding protein 1, Amacobpl gene

\section{Background}

Anopheles maculipennis complex include the historic vectors of malaria in Holarctic regions and can cause serious public health problems worldwide [1-3]. In this complex, there are several sibling species, among which An. maculipennis sensu stricto (s.s.) is the dominant one in Europe and the Middle East regions [4-6]. These

\footnotetext{
*Correspondence: Ghavami@zums.ac.ir

${ }^{1}$ Department of Medical Entomology and Vector Control, School of Medicine, Zanjan University of Medical Sciences, Zanjan, Iran Full list of author information is available at the end of the article
}

species occur partially sympatric and exhibit different behaviours in their host preference and selection of resting and breeding places [5-7]. Adults of these species are frequently found in human dwellings and animal shelters. They rest both indoor and outdoor shelters, and actively bite humans and animals [6-9]. Recently, various vector control methods have been developed against malaria vectors, such as An. maculipennis, and the most widely used method is insecticide application [10]. However, over the years, it has been reported that these species are resistant to some insecticides, such as DDT, organophosphate, carbamate, and pyrethroid [11, 12]. Accordingly, 
new mosquito-centered strategies for controlling and eliminating vector-borne diseases are urgently needed.

Repellents (impregnated bed nets and other formulations) seem to be applicable and efficient for the personal protection against biting insects and the diseases that they transmit to the inhabitants of endemic areas and travellers $[10,13]$. These compounds interfere in the olfactory system of insects, thereby preventing the transmission and spread of vector-borne diseases [14, 15]. Knowledge about the molecular elements of the mosquito odorant detection system and their specific functions is crucial for developing novel and eco-friendly methods for mosquito control [16]. Although numerous studies have contributed to understanding structure of various genes and their application in differentiation of species in Maculipennis Group [5, 17-20], there is still lack of information on the olfactory system of these species. This information may provide new insight into understanding the mechanism governing the speciation within the aforesaid complex and separation of their ecotypes.

Odorant-binding proteins (OBPs) are the most important agents in the olfactory system. These proteins play key roles in odorant perception and transmission to receptor sites, signal transduction, and triggering a series of behavioural responses $[14,15]$. The insect OBPs are soluble and globular peptides $(15-40 \mathrm{kDa}$, ca $120-150$ amino acids) with six highly cysteine-rich (Cys) residues, paired in three interlocked disulfide bridges [21, 22]. The affinities of these peptides for binding to ligand are $\mathrm{pH}$-dependent [23]. The hydrogen bonds involving in the $\mathrm{C}$-terminal loop break at low $\mathrm{pH}$ and open the loop, thus releasing further ligands [23-25]. These proteins are grouped into the following categories: classic, minus-C, plus-C, and atypical OBPs, based on the number of conserved Cys residues and their molecular structure [26]. Classic OBPs is the most important OBP in insects and contains six conserved cysteines having distinct disulfide connectives [26-28].

Among the classic group of the mentioned proteins, OBP1 is expressed exclusively in the head and olfactory organs of adult insects [29-31]. Meanwhile, the sexuallybiased expression of this protein is exhibited in different species of insects [27, 29-34]. Moreover, this protein is expressed rhythmically, and its transcription level is reduced after blood feeding by less than twofold in mosquitoes and other blood-feeding insects [34, 35]. The three-dimensional structure of Anopheles gambiae OBP1 (AgamOBP1) has already been detected, and indole and DEET have been identified as its ligands [36-38]. The homolog to AgamOBP1 in Anopheles stephensi has also been found as AsteOBP1, and its expression has revealed that its transcription level is sevenfold higher in female's than in male's antenna [34]. The amino acid sequence of exon 1 in this gene has been suggested as a molecular marker for diagnosis of biological forms in An. stephensi [39]. Moreover, based on the nucleotide sequence variation of intron I Asteobp1 gene in different populations, An. stephensi may prove to be a complex species [40].

Owing to the wide variation in the biological and ecological characteristics (host preference and vector competence.) within the members of Maculipennis Group, this study attempted to characterize, for the first time, the An. maculipennis OBP1 gene (Amacobp1), as a basic information for further studies to distinguish different taxa belonging to Maculipennis Group in the world. The present study also provides a new insight into the role of OBPs in the behaviour of different species and understanding speciation-related events within this complex.

\section{Methods}

\section{Mosquito collection}

Adults of An. maculipennis were collected by hand

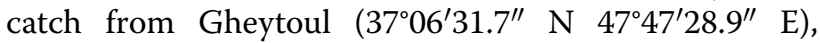
Ghara Bouta $\left(37^{\circ} 03^{\prime} 31.3^{\prime \prime}\right.$ N $47^{\circ} 46^{\prime} 09.5^{\prime \prime}$ E), Moshampa $\left(36^{\circ} 56^{\prime} 33.8^{\prime \prime} \mathrm{N} 47^{\circ} 40^{\prime} 28.8^{\prime \prime} \mathrm{E}\right)$, Kheyrabad (36 $55^{\prime} 25.4^{\prime \prime} \mathrm{N}$ $47^{\circ} 22^{\prime} 50.6^{\prime \prime}$ E), and Sari Aghol ( $36^{\circ} 49^{\prime} 23.5^{\prime \prime} \mathrm{N} 47^{\circ} 37^{\prime} 46.8^{\prime \prime}$ E) in Zanjan Province, the northwest of Iran, in summer 2016.

\section{Processing of mosquitoes}

Samples of female mosquitoes were selected for molecular survey. Identification of An. maculipennis s.s. were carried out using morphological characters $[3,7]$ and PCR-RFLP assay, based on ITS2 ribosomal DNA [20]. To extract genomic DNA (gDNA), the samples were stored at $-70{ }^{\circ} \mathrm{C}$ for $1 \mathrm{~h}$. Then the frozen samples were placed individually in 1.5- $\mathrm{ml}$ tubes and homogenized by an electrical homogenizer in a $400-\mu \mathrm{l}$ TENS buffer $(100 \mathrm{mM}$ of Tris, pH 8.0, $10 \mathrm{mM}$ of EDTA, $0.5 \mathrm{mM}$ of $\mathrm{NaCl}$, and $1 \%$ W/V SDS) containing a few glass beads. The gDNA of the samples was extracted as described by Ghavami et al. [20]. Briefly, $20 \mu \mathrm{g}$ of proteinase $\mathrm{K}$ was added to each sample, mixed gently and incubated at $55^{\circ} \mathrm{C}$ for $5 \mathrm{~h}$. After incubation, $100 \mu \mathrm{l}$ of $8 \mathrm{M}$ potassium acetate was added and kept on ice bath for $10 \mathrm{~min}$. The samples were then spun at $4000 \times \mathrm{g}$ for $10 \mathrm{~min}$, and the supernatants were transferred to new tubes. An equal volume of cold absolute ethanol was added to each tube, and the samples were spun at $8000 \times \mathrm{g}$ for $10 \mathrm{~min}$. Pellets of the samples were suspended in $0.5 \mathrm{ml}$ of $70 \%$ cold ethanol and eventually spun at $8000 \times \mathrm{g}$ for $10 \mathrm{~min}$. The final pellet was dried at room temperature and re-suspended in $50 \mu \mathrm{l}$ of TE buffer (10 mM Tris, $\mathrm{pH}$ 8.0, and 1 mM EDTA).

The PCR assays were conducted in two steps. In the first phase, the splicing region between the upstream 
Table 1 Sequences of nucleotides in different genes of encoded odorant-binding protein 1 (OBP1) in Anopheles species used for designing the degenerate primers in this study

\begin{tabular}{lll}
\hline Anopheles species & Gene code $^{\text {a }}$ & Sequence location \\
\hline An. culicifacies & ACUA014299 & KI424042: 15893-17790 \\
An. dirus & ADIR008409 & KB672813: 2498551-2500404 \\
An. epiroticus & AEPI007538 & KB671606: 54036-56043 \\
An. farauti & AFAF003898 & KI915040: 6703195-6705104 \\
An. funestus & AFUN008615 & KB668715: 290624-291834 \\
An.gambiae & AGAP029062 & 2R: 35643035-35644609 \\
An.melas & AMEC013481 & KI920369: 35115-36111 \\
An.merus & AMEM011461 & KI915244: 175565-177040 \\
An. quadriannulatus & AQUA010363 & KB665843: 2529565-2530556 \\
An. sinensis & ASIS018762 & KI916356: 352496-353910 \\
An. stephensi & ASTE010148 & KB664334: 168200-170200 \\
\hline
\end{tabular}

${ }^{a}$ Gene numbers submitted to the VectorBase (https://www.vectorbase.org)

Table 2 Details of the primers used in this study

\begin{tabular}{ll}
\hline Primer name & Nucleotide sequence $\left(\mathbf{5}^{\prime} \boldsymbol{\rightarrow} \mathbf{3}^{\prime}\right)$ \\
\hline AnFa & TTGTCTCDTGATTGATTTGTCA \\
AnRa & CTTYTCGTCYTCGTGGATTCT \\
AnFb & AGATCCACGARGACGARAAG \\
AnRb & GAAAAATACGGTCTGATTATAG \\
AmaF & GTGTGGATAGTTCTTGGAACGG \\
AmaR & AAGAAATGCATCGCACACTCGC
\end{tabular}

$R=A+G, Y=C+T, D=A+G$

and the downstream of deduced gene was amplified by degenerate primers. These primers were designed from the conserved regions of this gene in known anopheline species (Table 1) by means of BioEdit version 7 software [41]. These primers are not specific and could be used to generate the OBP1 gene in different anopheline species. The first stage of PCR was performed from mosquito gDNA in two separated ' $a$ ' and ' $b$ ' reactions. Each $50 \mu \mathrm{l}$ of PCR reaction of this stage contained $4 \mu \mathrm{g}$ of gDNA as the template, $1.5 \mathrm{mM}$ of $\mathrm{MgCl}_{2}, 0.2 \mathrm{mM}$ of dNTPs (SinaClon, Iran), $10 \mathrm{pM}$ of each primer (AnFa, AnRa and AnFb, AnRb; Table 2), and $1 \mathrm{U}$ Taq of DNA polymerase (SinaClon, Iran). Thermal profile of PCR reaction was started with one cycle of initial denaturation at $95^{\circ} \mathrm{C}$ for $3 \mathrm{~min}$, followed by running through 30 cycles of $60 \mathrm{~s}$ at $94{ }^{\circ} \mathrm{C}$, $60 \mathrm{~s}$ at $68{ }^{\circ} \mathrm{C}$, and $90 \mathrm{~s}$ at $72{ }^{\circ} \mathrm{C}$. The final extension was performed in one cycle at $72{ }^{\circ} \mathrm{C}$ for $5 \mathrm{~min}$.

The second phase of PCR was performed by using the specific primers, AmaF and AmaR (Table 2). These primers, specific to An. maculipennis s.s., were designed based on the nucleotide sequence of the first PCR products. The forward primer (AmaF) was located around the translation start codon and the reverse primer (AmaR) at the translation stop of the Amacobp1 gene. For each $50 \mu \mathrm{l}$ of PCR reaction, $10 \mathrm{ng}$ of gDNA was amplified with $10 \mathrm{pM}$ of each primer using the following conditions: $95^{\circ} \mathrm{C}$ for $5 \mathrm{~min}$ in one cycle, $93^{\circ} \mathrm{C}$ for $30 \mathrm{~s}, 60^{\circ} \mathrm{C}$ for $50 \mathrm{~s}$, and $72{ }^{\circ} \mathrm{C}$ for $2 \mathrm{~min}$. This cycle was repeated 35 times.

Each PCR run contained field samples and a negative control (molecular grade water instead of DNA template). Aliquots of the amplicons were analysed by electrophoresis on $1.5 \%$ agarose gel stained with safe stain (SinaClon, Iran) and visualized under a UV light. The amplified fragments were purified by PCR Clean-Up Kit (SinaClon, Iran) and sequenced bi-directionally by SeqLab GmbH (WWW.Seqlab.De) using the forward and reverse specific primers. The nucleotide sequences of the samples were aligned and edited by the aid of BioEdit and Sequin software (https://www.ncbi.nlm.nih.gov/ Sequin). The Amacobp1 nucleotide sequences in studied populations were submitted to the GenBank and are available with accession nos. MG209518-MG209522 and MN080505-MN080524.

The consensus of nucleotide and amino acid sequences were achieved by Basic Local Alignment Search Tool (BLAST) (https://blast.ncbi.nlm.nih.gov/Blast.cgi), BLASTn and P BLAST, which were searched for fragment identification and compared with other submitted relevant sequences in NCBI (https://www.ncbi.nlm.nih. gov), VectorBase (https://www.vectorbase.org) and FlyBase (http://www.flybase.org).

The mature peptide sequences identified in this study and in the previous studies were aligned using Clustal Omega (https://www.ebi.ac.uk/Tools/msa/clustalo/). The alignments were used to construct phylogenetic trees using the Molecular Evolutionary Genetics Analysis software version 6 (MEGA6) [42]. The final unrooted consensus trees were generated with 1000 bootstrap trials using the Neighbor-Joining, Maximum Likelihood, and Minimum-Evolution methods with a cut-off bootstrap value of 50 and using $\mathrm{p}$-distance model.

\section{Results}

The fragments of 1100 and a 1200 bp were amplified in the ' $a$ ' and ' $b$ ' section of the first PCR step, respectively, in 200 specimens (40 samples from each population) by degenerated primers. In the second step, a 1261-bp fragment was obtained in studied samples by specific primers (Fig. 1). A total of 20 PCR products of specific primers (4 samples from each population) and 5 PCR products of degenerated primers (one sample from each population) were selected randomly for sequencing. Analysing the sequences of degenerated primer products revealed that the sequence of Amacobp1 gene has 1341 nucleotides. This analysis also exhibited the existence of two 


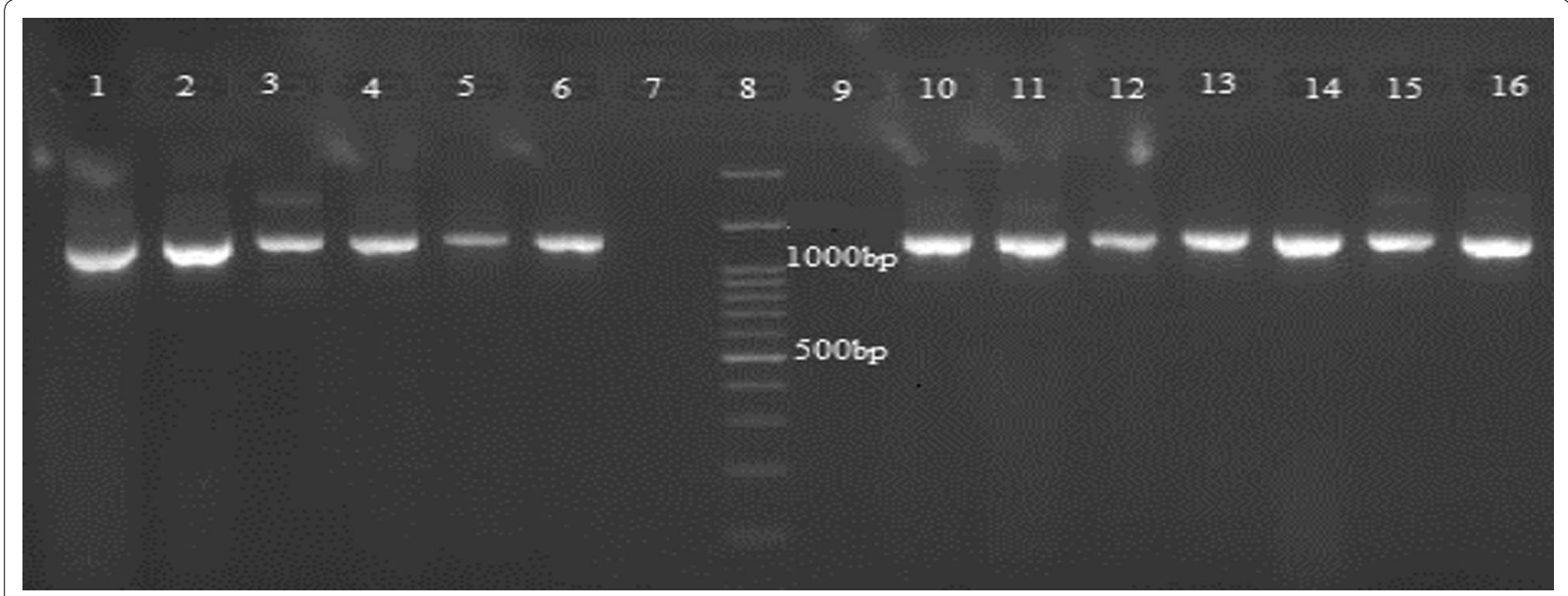

Fig. 1 PCR amplification of odorant-binding protein 1 fragment in An. maculipennis s. s. Lanes 1-3 and 4-6, the first and the second amplicons produced by degenerated primers, respectively; lanes 7 and 9, negative controls; lane 8, 100 bp DNA marker; lanes 10-16, products of specific primers

introns and three $\left(5^{\prime}\right.$, internal, and $\left.3^{\prime}\right)$ exons in deduced gene (Fig. 2). In amplified fragments, the transcription start site (GTCGTCG indicated as +1 in Fig. 2) occurred in 57 nucleotide upstream of the start codon (ATG). However, a TCAGG sequence, similar to the arthropod imitator, was observed in 15 nucleotide upstream of the transcription start site. The Goldberg-Hogness box, known as TATA box, is located in the 35-bp upstream of the predicted transcription start site. Imperfect polyadenylation signal sequences AATA, AATTAA, AATTT, and AAATAA were found in the 155, 161, 204, and 211 downstream of the poly ( $\mathrm{dA})$ tail, respectively. The position of transcription start site, the start and stop codons, and the open reading frame (ORF) was interrupted by two introns, and the putative polyadenylation signals are all shown in Fig. 2.

The processed transcript was $715 \mathrm{bp}$ long with an approximately 57 bp $5^{\prime}$ UTR and 224 bp $3^{\prime}$ UTR. The coding region contained $434 \mathrm{bp}$ long. In this region, three exons, including $5^{\prime}$, internal, and $3^{\prime}$ of 160,256 , and $18 \mathrm{bp}$, respectively, encoded 144 amino acids of OBP1. The sizes of intron I and intron II in the deduced gene were 268 and 358 nucleotides, respectively.

Twenty fragments comprising $5^{\prime}$ UTR, ORF, and partial $3^{\prime}$ UTR of Anmacobp1 gene were generated by specific primers. The alignment was $1261 \mathrm{bp}$ in length (including primers $44 \mathrm{bp}$ ), and 41 sites (3\%) were variable among the sequences. Each of $5^{\prime}$ and internal exons had three variable nucleotide sites, and the nucleotide polymorphism in introns I and II were in 12 and 10 sites, respectively. The percentages of nucleotide polymorphism in coding and noncoding regions of the deduced gene were $1.4 \%$ and $3.8 \%$, respectively. All the single nucleotide polymorphisms in the coding region were silent. Multiple alignments of these sequences in representative populations are illustrated in Fig. 3.

Standard protein BLAST analysis of $A m a c O B P 1$ indicated that the overall structure of this peptide was highly similar to Anopheles atroparvus OBP1, which was annotated in VectorBase with AATE020811-RA code. The analysis also showed that $A m a c O B P 1$ alignment shared $87 \%$ amino acid similarity with Anopheles sinensis (AIR09579), as well as $86 \%$ identity with An. gambiae (AAI84179), 83\% with An. stephensi (ACS83757), and 83\% with Drosophila melanogaster (FBpp0078305). Comparison of amino acid sequences of $A m a c O B P 1$ with their homologous in $A n$. atroparvus revealed that they had only one non-identical amino acid. In An. maculipennis, cysteine $(C)$ had been replaced by glycine $(G)(C \rightarrow G)$ in locus 5 (Fig. 4).

Pairwise comparison of amino acids in OBP1 among different species of Anopheles showed the existence of six conserved $\mathrm{C}$ amino acids, five of which were in the first exon, and the remaining one in the second exon. This analysis also suggested that the two exons (II and III) were relatively conserved between anopheline species both in terms of their sequence and length. Comparing amino acid sequence of $A m a c O B P 1$ with AgamOBP1 indicated that the $\mathrm{C}$-terminal of $A m a c O B P 1$ has a high similarity $(95 \%, 3 / 60)$ to that of AgamOBP1.

The comparison between the N-terminal of Ama$c O B P 1$ and its orthologous polypeptide in different species revealed that this region was less conserved, and 19 amino acids of this part were signal peptides and 
a

$-373$

$-303$

$-223$

$-163$

$-93$

$-23$

47

117

187

257

327

397

467

537

607

677

747

817

887

957

1027

1097

1167

1237

1307
CTGCGTCGTTCGGGTTCTTTTCCGAGCTGCTGCATTCGGTTGGGGCGCACATTGTTTTCCTTGAGGTTTT TGTTTTCCCTACCTTCCGGAACTGATCCGGCGGAAGCTTGTTCTCAATTTATTCTTTCTTTTTTTTCCAG CATTTGCTTTTTCGCAGACCCACCAGAATTAATTAACGATTCGTCGTGGGCCAGTCATTGTCGCCTACCG TTTAATTATCGGTCAACAGAAACGGGAAGATAATTGGCTAGGGATTCATCGTTGTGGGTCGCGGGGTGGC ATGTGTGTGGATAGTTCTTGGAACGGTTCTCGGA GCCGCCAATCGGGACGTGGGAAGC TATAAAAATGAA CTGAAATG TCAGGTGCAGA GTCA GTCGTCGCCCTCGCTACTCGACTGTAACTTGTTGTAGACTTTTTGAA AAGGGAAAAACATGTTATTGCTTGGTTACATTTGTGCCGCTATTGGCCTTTTCTCTATGGTAATCGCCGA

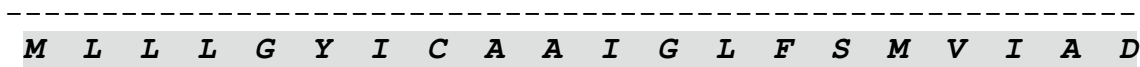
CACTACGCCTCGCCGTGATGCAGAATATCCTCCGCCGGAGCTGCTCGAAGCCTTGAAACCACTGCACGAT

-
$\begin{array}{llllllllllllllllll} & \end{array}$

$\begin{array}{lllllll}\text { V } & \text { C V G K T G V T N }\end{array}$

TGTTTGTAGTTCTTACCTCTGCCCTAAATGTGGAAAAGGATCATACTTTTTTTTATTACCGTTACCAACT TATTAAGAATGGTTACATTTGTGGTTAACAGCCAGCTTGAATTAATTTTAAATTTTTCGTTCTTCTTTTT AAAAATTAATTTTAAAAGCCCGGAAAAAAAAAGATTTTTTGACGTGGAATAACCTCCTAATCCCTGACAC ACTGGTCCACTTTCATAGAAGCCATCAAGAAATTCAGCGATGAAGAGATCCACGAGGACGAAAAGCTCAA

$\begin{array}{llllllllllllllllll}E & A & I & K & K & F & S & D & E & E & I & H & E & D & E & K & L & K\end{array}$
GTGCTACATGAACTGTCTGTTCCACGAGGCGAAGGTGGTCGACGACAACGGGGACGTGCACCTGGAGAAG $\begin{array}{cllllllllllllllllllllll}C & Y & M & N & C & L & F & H & E & A & K & V & V & D & D & N & G & D & V & H & L & E & K\end{array}$ CTGCACGCCTCGCTCCCGGACTCGATGCACGACATCGCGATGCACATGGGCAAGCGCTGCCTCTACCCGG

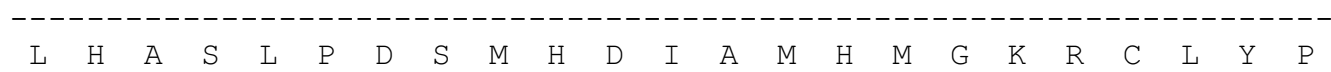
AGGTCGAGAATCTCTGCGACAAGGCGTTCTGGCTGCACAAGTGCTGGAAGCAGTCCGACCCGAAGGTAAC -------------------------------------------------------------> $\begin{array}{llllllllllllllllllllll}\mathrm{E} & \mathrm{V} & \mathrm{E} & \mathrm{N} & \mathrm{L} & \mathrm{C} & \mathrm{D} & \mathrm{K} & \mathrm{A} & \mathrm{F} & \mathrm{W} & \mathrm{L} & \mathrm{H} & \mathrm{K} & \mathrm{C} & \mathrm{W} & \mathrm{K} & \mathrm{Q} & \mathrm{S} & \mathrm{D} & \mathrm{P} & \mathrm{K}\end{array}$ TGGAGAAATGCGAACCGATTCTCCGCGCGTTTCGCGCTTCCCACCGATTTGCCGGCAGCCAGCCAACCAG CCAGCCTGCGCCGGCCGGTAGTTGATCGACCCCGCGCGATTCGCAATAATTTCTTTACGGCGGCAAGTTT TTAATTGAAAAAGTTTAACTTTTAATTGCTTCCGGCAACCGTGCCACCGAGACGGTCTGATACCGGCGGA AAATATGACCCGCCGACTCGACGAAGATGCACCGCGGTTCGCCAGCGCTCACTCCGGGAAAGGCGGCGAT GGATTCGGAATTAAAATTCATTCATTCGACTTTTCCCTTTTCTTGACCCACTTTTCGTCCTTTCTTTCGC AGCACTATTTCCTAGTATAAGCGGCTCCGTTTTGTTGCCGGAAAAAGCGAGGTGCGAGTGTGCGATGCAT

$$
--------------->
$$

$$
\begin{array}{llllll}
H & Y & F & L & V
\end{array}
$$

TTCTTCATGATCGGCCACCGGATGACGAGACGACGACCGCCGGGTCTCGGTCAACTGTAAGCCGCTCCAC CAAACGCCACGGTCATCAGGCATCAACGCGCGCGCCCTTCGGAATAGT AATTAACCTGTTTCTTTTTCTC CCTCGACTGCACCGCACGTAGT AATTTGCAAATAAAAAAA

b

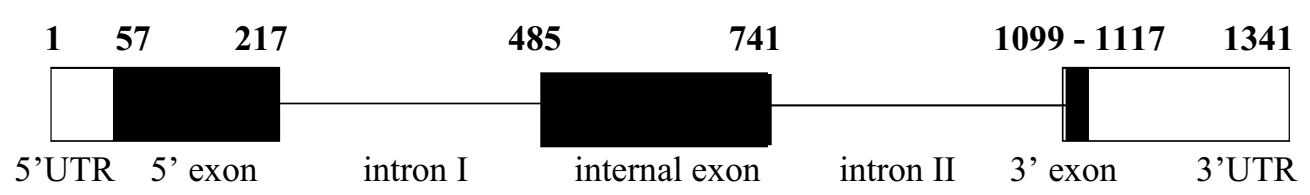

Fig. 2 Sequence and schematic structure of An. maculipennis odorant-binding protein 1 (Amacobp 1) gene. a Genomic sequence of Amacobp 1 gene containing two introns among three exons. The deduced amino acids sequences of Amacobpl gene is shown below the coding regions. Start (ATG) and stop (TAA) codons are italicized, in bold. A putative imitator sequence, TCAGG, similar to the arthropod imitator consensus, the consensus for TATA box/TATAAA, and the imperfect polyadenylation signals (AATA, AATTAA, AATTT, AAATAA) are shown in bold-italic text. Solid underlines indicate untranslated reading fragments (UTRs), and dashed underlines show the open reading fragments (ORFs) of exons. The signal peptide sequence is highlighted in gray and italicized. $\mathbf{b}$ Schematic structure of Amacobpl gene. $5^{\prime}$ exon, internal exon, and $3^{\prime}$ exon are indicated in boxes. Boxes with solid black show ORFs. They are connected with lines that indicate the introns. The $5^{\prime}$ and $3^{\prime} U$ TRs are shown in hallow boxes. Numbers indicate the relative positions of UTRs, ORFs, and intron regions 


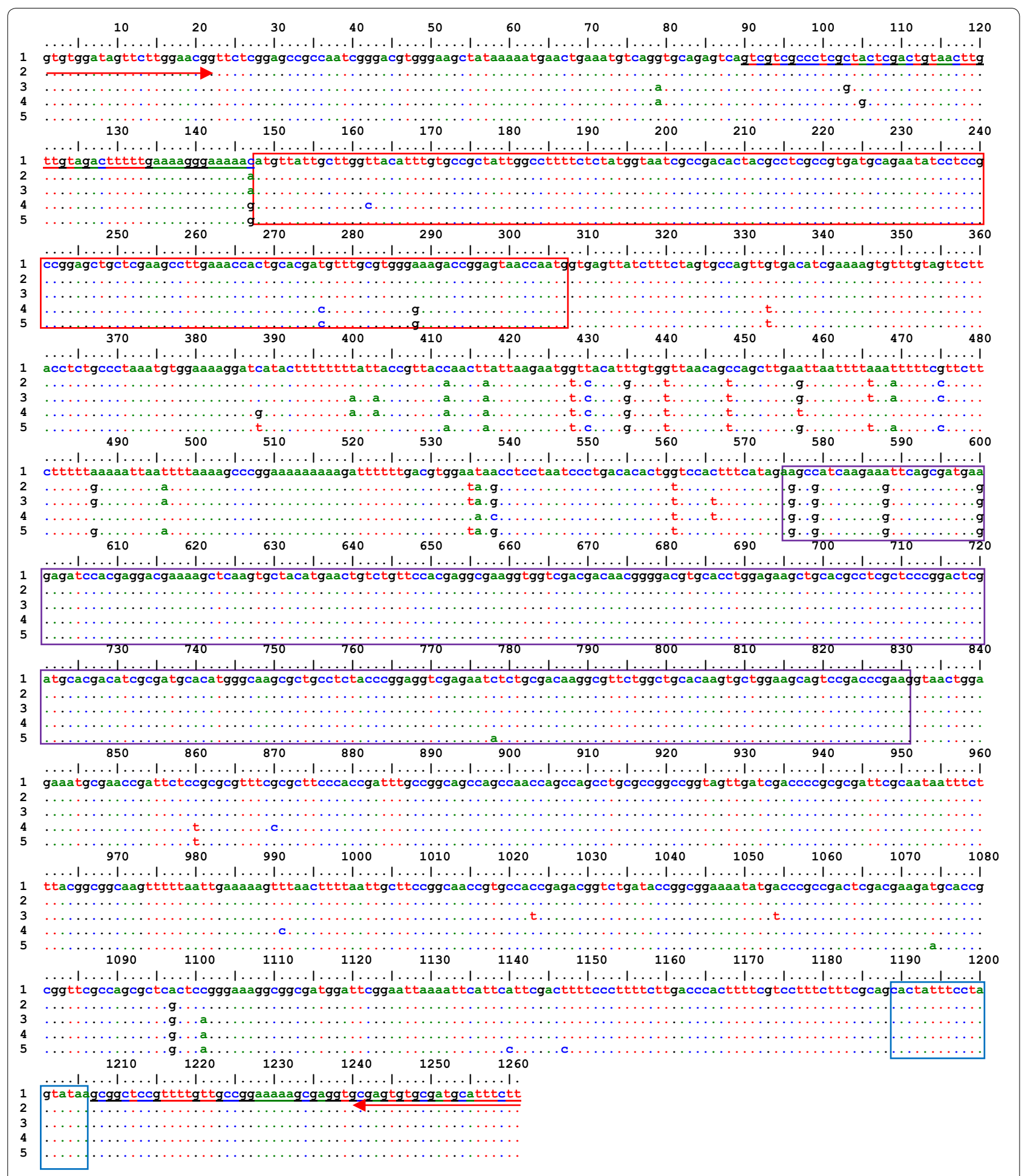

Fig. 3 Nucleotide polymorphism and schematic structure of amplified fragment of Amacobpl gene in representative populations of An. maculipennis s.s. (based on 5 collected sites) with specific primers. Red arrows, indicate the position of specific primers. Horizontal numbers, indicate the study populations; 1: Kheyrabad (MG209521); 2: Ghara Bouta (MG209519); 3: Gheytoul (MG209520); 4: Sari Aghol (MG209518) and 5: Moshampa, (MG209522). UTRs are mentioned with underline letters, and 5' exon, internal exon, and $3^{\prime}$ exon are shown in red, violet and blue boxes, respectively 


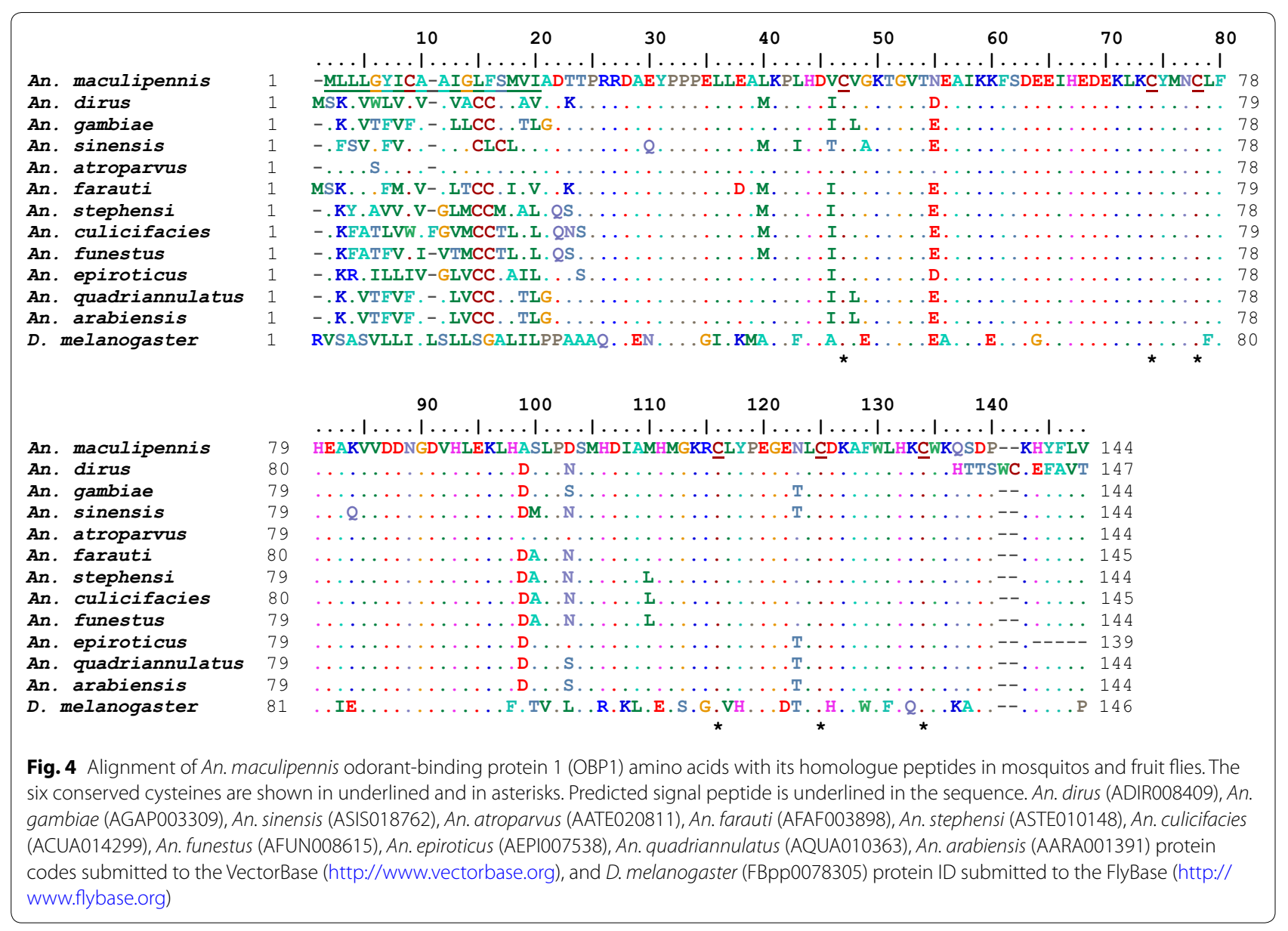

had specific sequencing. However, C-terminal of the $A m a c O B P 1$ polypeptide had a high level of conservation between the species and exon III of deduced $o b p 1$ gene in An. maculipennis encoding a short-chain amino acid peptide ( 6 amino acids), which its composition was similar to that of other anopheline species.

The phylogenic relationship and topology based on the amino acid of polypeptide in deduced protein, along with sequences available in the VectorBase and FlyBase, is illustrated in Fig. 5. The Neighbour-Joining, Minimum Evolution, and Maximum likelihood analyses showed these OBPs cluster in three groups. An. atroparus and $A n$. sinensis were the closest species to $A n$. maculipennis. This clade, together with Anopheles dirus and Anopheles farauti, was deposited in one group. Anopheles farauti, An. dirus, An. stephensi, Anopheles funestus, and Anopheles culicifacies were sister groups with An. maculipennis. The remaining species, including An. gambiae, Anopheles quadrimaculatus, and Anopheles arabiensis, which were initially separated from anopheline species, were located in the third group (Fig. 5).

\section{Discussion}

Anopheles maculipennis s.s. is the dominant anopheline species of the Maculipennis Group in Europe and the Middle East regions [4-9]. There has previously been no available information regarding Amacobp gene and sequence details of olfaction-associated genes in this species. Therefore, generating relevant genetic data was a research priority for investigations focusing on olfactory gene functions in different species of Maculipennis Group.

Using degenerate primers in this study, the fragment sequences of $1100 \mathrm{bp}$ within gDNA, a region covering the obp1 gene in An. maculipennis, were amplified by PCR, and, thus, these primers could be suggested for use in studying obp 1 gene in different Anopheles species. Applying degenerate primes to amplify the candidate $o b p 1$ gene from gDNA is a convenient way to identify the molecular characters. Nonetheless, this method might miscalculate the data related to $5^{\prime}$ and $3^{\prime}$ UTRs of the gene. Hence, further studies are necessary to obtain the full-length cDNA clones from mRNA by RACE-PCR methods. 


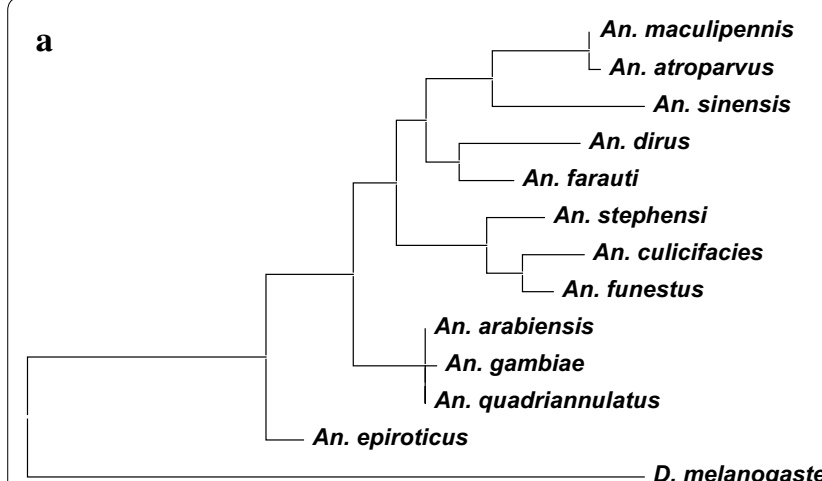

0.05

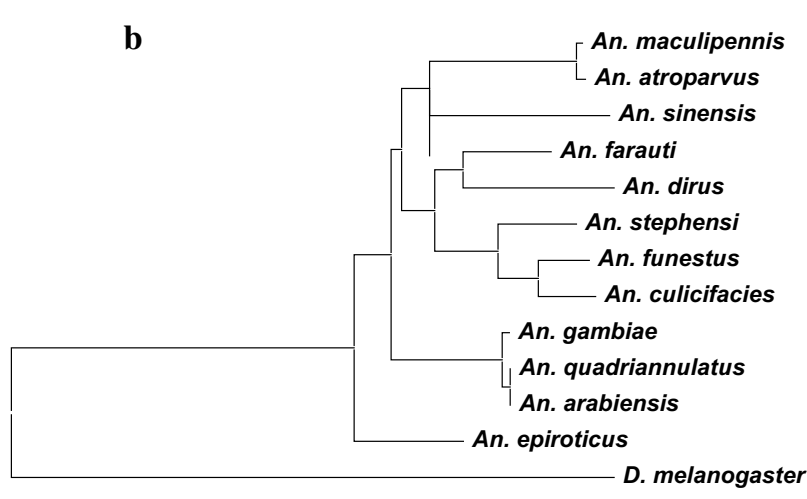

0.05

Fig. 5 Phylogenetic tree relationship between An. maculipennis odorant-binding protein 1 (OBP1) with its homologues in An. atroparvus (AATE020811), An. sinensis (ASIS018762), An. dirus (ADIR008409), An. farauti (AFAF003898), An. stephensi (ASTE010148), An. culicifacies (ACUA014299), An. funestus (AFUN008615), An. arabiensis (AARA001391), An. gambiae (AGAP003309), An. quadriannulatus (AQUA010363), An. epiroticus (AEPI007538) protein codes submitted to the VectorBase (http://www.vectorbase.org), and D. melanogaster (FBpp0078305) protein ID submitted to the FlyBase (http://www.flybase.org). a Maximum likelihood; b neighbor-joining and minimum-evolution

In the present study, analysis of amplified fragments, products with specific primers, showed that the ORF of Amacobp1 gene has 1060 nucleotides, and this region encodes a 144-amino-acid polypeptide. Comparison of the amplified fragments in studied samples revealed that the AmacOBP1 is conserved within the population level. To the best of our knowledge, this is the first report describing the Amacobp1 gene. The generated information could be useful for the development of molecular techniques in studying Amacobp1 expression patterns in different olfactory and gustatory organs.

Comparison of amino acid sequencing of AmacOBP1 with its orthologous peptides demonstrated that this protein has $86 \%$ similarity with AgamOBP1. Previous studies have also indicated that AgamOBP1 displays high affinity with its ligands. The C-terminal loop of this peptide covers the binding cavity, and attractant and repellent have been identified as two of its ligands [36, 37]. The alignment of $A m a c O B P 1$ with AgamOBP1 and other mosquito orthologous peptides showed a high level of conservation in the overall protein structure. The availability of the primary and the secondary structures of $A m a c O B P 1$ would help prospective modelling studies that compare and contrast the two anopheline OBP1 proteins. Therefore, future studies are suggested to investigate the expression profile and ligand-binding feature in AmacOBP1. Moreover, identifying key amino acids in C-termini and detecting main hydrophobic, polar and nonpolar residues in signal peptides that involve in the ligand binding could be the other subjects for future investigations.

By comparing amino acid sequences in the C-terminal of $A m a c O B P 1$ with its orthologous peptides a high identity (95\%) was found between AmacOBP1 and AgamOBP1. DEET and other repellents have been demonstrated to target OBP function by disrupting interaction with natural ligands to prevent connection with other compounds of the olfactory signaling pathway. Although effective, DEET has been recognized to be toxic, to have reduced efficacy with time after application, and to be ineffective against developed resistance species [43, 44]. Therefore, the need for a new, more efficient and less toxic repellent is highly felt. In silico ligand-binding studies in future may be valuable for screening mosquito repellents. In addition, X-ray crystallography and nuclear magnetic resonance (NMR) have successfully been applied to solve the structure of OBP from different insect species [24]. Comparative structure of $A m a c O B P 1$ is certainly important in forthcoming research, particularly to address questions regarding molecular feature of the pH-dependent conformation changes (Additional file 1 ).

The phylogeny analysis in the present study showed a great variety in the amino acid alignment of OBP1 peptides in different Anopheles species, and AmacOBP1 exhibited the highest amino acid variation among these peptides. Considering these results, it is suggested that the OBP1-encoding gene in the anopheline is derived from the same ancestor and diverged after speciation. On the other hand, the AmacOBP1, which has the most variation, may have a long history or high rate of evolution in adaptation to ecological conditions. The high rate of variation within $A m a c O B P 1$ may suggest the ongoing evolutionary mechanisms, including genetic drift and gene flow that are still faster in the speciation within this 
complex. This observation may be explained in further studies by the wide variation and distribution patterns of different members in the Maculipennis Group.

The present study showed that the N-terminal of AmacOBP1 assets signal peptides. Despite the consistency of amino acid sequences in C-terminal, this part has specific sequence and little sequence conservation. In the context of evolution, the pressures that shaped the properties of the mature OBP proteins are fundamentally different from those that shaped their signal peptides. The mature proteins evolve to interact with external chemical signals and to function in a behavioural context, while the signal peptides develop to interact with intracellular signals involved in trafficking. All of these OBPs are postulated to be expressed in and secreted by glia-like support cells that ensheathe the sensory neuron somata and have highly folded apical membranes facing the sensillum lumen. The OBPs are presumably secreted across these apical membranes. The OBP signal peptides contain the hydrophobic cores of 10-12 amino acids flanked by more hydrophilic regions. These characteristics of signal peptides are involved in protein translocation into endoplasmic reticulum for the purpose of secretion $[45,46]$. In the same general cell type, these intracellular signals are expected to be conserved, especially within a given mosquito species, and are poorly conserved between species. This class of conservation suggests that OBP1 signal peptides might be involved in class-specific processing for expressed homologous proteins. Therefore, they could be used as a molecular marker for studying the population structure of species in Maculipennis Group. Moreover, signal peptides are appropriate sequences to design specific primers and probes for studying the gene expression pattern in olfactory and gustatory organs of An. maculipennis by qPCR assay in different populations.

\section{Conclusion}

Degenerate primers in this research are suggested for study on obp1 gene in different Anopheles species. Prospective studies are recommended to investigate the gene expression profile and ligand-binding profiles in Ama$c O B P 1$. Further investigations are also suggested to identify the key amino acids, main hydrophobic and polar/ nonpolar residues of $A m a c O B P 1 \mathrm{C}$-terminal in ligandbinding. The signal peptide in $\mathrm{N}$-terminal of AmacOBP1 is advised for designing specific primers and probes, to detect the expression pattern of this gene in the main olfactory and gustatory organs for assessing the molecular mechanisms and developing novel repellents against this vector in future surveys. In addition, this peptide is proposed as a molecular marker for detection of $A n$. maculipennis intraspecific ecotypes and diagnosis of different species within Maculipennis Group.

\section{Supplementary information}

Supplementary information accompanies this paper at https://doi. org/10.1186/s12936-019-3058-6.

Additional file 1: Appendix 1. Nucleotide polymorphism and schematic structure of amplified fragment of Amacobp 1 gene in 25 samples of

studied populations of An. maculipennis s. S.

\section{Abbreviations}

AmacOBP1: Anopheles maculipennis odorant-binding protein 1; BLAST: basic local alignment search tool; BLASTn: basic local alignment search tool for nucleotides; bp: base pair; DDT: dichlorodiphenyltrichloroethane; DEET: N, $\mathrm{N}$-diethyl-m-toluamide; gDNA: genomic DNA; ITS2: internal transcript spacer 2; KD: Kilo Dalton; MEGA6: molecular evolutionary genetics analysis version 6; mRNA: messenger ribonucleic acid; NCBI: The National Center for Biotechnology Information; OBPs: odorant-binding proteins; ORF: open reading frame; P BLAST: basic local alignment search tool for peptides; PCR: polymerase chain reaction; RACE: rapid amplification of CDNA ends; RFLP: restriction fragment length polymorphism; s.s.: sensu stricto; UTR: untranslated region.

\section{Acknowledgements}

The authors express their thanks to Mr. Taghiloo, Mohammadi, and Torabi for their collaboration in the collection of samples. We duly acknowledge the financial support of this project provided by Deputy of Research and Technology of Zanjan University of Medical Sciences, Iran. We also would like to thank to Ms. Safari and anonymous reviewers for their comments that improved the quality of the manuscript.

\section{Authors' contributions}

Conceived and designed the experiment: MBG and SK. Analysed the data: MBG. Contributed reagents/materials/analysis tools: MBG and SK. Wrote the paper: MBG, NDD, and SK. All authors read and approved the final manuscript.

\section{Funding}

This study was supported by Zanjan University of Medical Sciences, Zanjan, Iran (Number A-11-84-10).

Availability of data and materials

Data supporting of this article is included within the article and additional file.

Ethics approval and consent to participate

Not applicable.

Competing interests

The authors declare that they have no competing interest.

Consent for publication

Not applicable.

\section{Author details}

${ }^{1}$ Department of Medical Entomology and Vector Control, School of Medicine, Zanjan University of Medical Sciences, Zanjan, Iran. ${ }^{2}$ Malaria and Vector Research Group, Pasteur Institute of Iran, Tehran, Iran.

Received: 28 February 2019 Accepted: 8 December 2019 Published online: 17 January 2020

\section{References}

1. Jetten $\mathrm{TH}$, Takken W. Anophelism without malaria in Europe. A review of the ecology and distribution of the genus Anopheles in Europe. Netherlands: Wageningen Agricultural University Papers; 1994. p. 94-5.

2. Service MW, Townson H. The Anopheles vector. In: Warrell DA, Gilles HM, editors. Essential malariology. 4th ed. London: CRC Press; 2002.

3. Harbach RE. The classification of genus Anopheles (Diptera: Culicidae): a working hypothesis of phylogenetic relationships. Bull Entomol Res. 2004;94:537-53. 
4. Ramsdale C, Snow K. Distribution of the genus Anopheles in Europe. Eur Mosq Bull. 2000;7:1-26.

5. White GB. Systematic reappraisal of the Anopheles maculipennis complex. Mosq Syst. 1978;10:13-44.

6. Zahar AR. Vector bionomics in the epidemiology and control of malaria. Part II. The WHO European region and the WHO Eastern Mediterranean region, vol. 2. Geneva: World Health Organization; 1990.

7. Harbach RE. The phylogeny and classification of Anopheles. In: Manguin $\mathrm{S}$, Ed. Anopheles mosquitoes: New Insights into Malaria Vectors. InTech Open Science; 2013. p. 3-79 http://dx.doi.org/10.5772/3392.

8. Linton YM, Smith L, Koliopoulos G, Samanidou-Voyadjoglou A, Zounos AK, Harbach RE. Morphological and molecular characterization of Anopheles (Anopheles) maculipennis Meigen, type species of the genus and nominotypical member of the maculipennis complex. Syst Entomol. 2003;28:39-56.

9. Becker N, Petrić D, Boase C, Lane J, Zgomba M, Dahl C, Kaiser A. Mosquitoes and their control. London: Springer; 2010.

10. Devillers J. Use of insecticides indoors for the control of mosquitoes: exposure scenarios and modeling. In: Devillers J, editor. Computational design of chemicals for the control of mosquitoes and their diseases. Boca Raton: CRC Press; 2017. p. 423-52.

11. Akiner MM, Caglar SS, Simsek FM. Yearly changes of insecticide susceptibility and possible insecticide resistance mechanisms of Anopheles maculipennis Meigen (Diptera: Culicidae) in Turkey. Acta Trop. 2013;126:280-5.

12. Chavshin AR, Dabiri F, Vatandoost H, Mohammadi Bavani M. Susceptibility of Anopheles maculipennis to different classes of insecticides in West Azarbaijan province, northwestern Iran. Asian Pac J Trop Biomed. 2015;5:403-6.

13. Debboun M, Frances S, Strickman D. Insect repellents: principles, methods, and uses. New York: CRC Press; 2006.

14. Dickens JC, Bohbot JD. Mini review: mode of action of mosquito repellents. Pesticide Biochem Physiol. 2013;106:149-55.

15. Qiu YT, Van Loon JA. Olfactory physiology of blood-feeding vector mosquitoes. In: Takken W, Knols BGJ, editors. Ecology and control of vectorborne diseases: olfaction in vector-host interactions, vol. 2. Netherland: Wageningen Academic Publisher; 2010. p. 39-62.

16. Vijay V, Reji G. Herbal insecticides: repellents and biomedicines: effectiveness and commercialization. London: Springer; 2016.

17. Oshaghi MA, Sedaghat MM, Vatandoost H. Molecular characterization of the Anopheles maculipennis complex in the Islamic Republic of Iran. East Mediterr Health J. 2003;9:659-66.

18. Sedaghat MM, Linton Y-M, Oshaghi MA, Vatandoost $H$, Harbach RE. The Anopheles maculipennis complex (Diptera: Culicidae) in Iran: molecular characterization and recognition of a new species. Bull Entomol Res. 2003:93:527-35

19. Djadid ND, Gholizadeh S, Tafsiri E, Romi R, Gordeev M, Zakeri S. Molecular identification of Palearctic members of Anopheles maculipennis in northern Iran. Malar J. 2007;6:6.

20. Ghavami MB, Djadid ND, Haniloo A. Molecular characteristics of Anopheles maculipennis Meigen in Zanjan, northwest of Iran, inferred from ITS2 sequence analysis. Pak J Biol Sci. 2008;11:539-45.

21. Field LM, Pickett JA, Wadhams LJ. Molecular studies in insect olfaction. Insect Mol Biol. 2000;9:545-51.

22. Fan J, Francis F, Liu Y, Chen JL, Cheng DF. An overview of odorant-binding protein functions in insect peripheral olfactory reception. Genet Mol Res. 2011;10:3056-69.

23. Leite NR, Krogh R, Xu W, Ishida Y, Iulek J, Leal WS, Oliva G. Structure of an odorant-binding protein from the mosquito Aedes aegypti suggests a binding pocket covered by a pH-sensitive "Lid". PLoS ONE. 2009;26(4):e8006.

24. Wogulis M, Morgan T, Ishida Y, Leal WS, Wilson DK. The crystal structure of an odorant binding protein from Anopheles gambiae: evidence for a common ligand release mechanism. Biochem Biophys Res Commun. 2006:339:157-64

25. Pelosi P, lovinella I, Felicioli A, Dani FR. Soluble proteins of chemical communication: an overview across arthropods. Front Physiol. 2014:27(5):320.

26. Mastrobuoni G, Qiao H, lovinella I, Sagona S, Niccolini A, Boscaro F, et al. A proteomic investigation of soluble olfactory proteins in Anopheles gambiae. PLoS ONE. 2013;25(8):e75162.
27. Zhou JJ, He XL, Pickett JA, Field LM. Identification of odorant-binding proteins of the yellow fever mosquito Aedes aegypti: genome annotation and comparative analyses. Insect Mol Biol. 2008;17:147-63.

28. Pelletier J, Leal WS. Genome analysis and expression patterns of odorantbinding proteins from the southern house mosquito Culex pipiens quinquefasciatus. PLoS ONE. 2009;16(4):e6237.

29. Zhao H, Zhao W, Gao P, Zhang G, Jiang Y. Sequence and expression characterization of an OBP1 gene in the Asian honeybee, Apis cerana cerana (Hymenoptera: Apidae). Appl Entomol Zool. 2014;49:189-96.

30. Biessmann H, Walter MF, Dimitratos S, Woods D. Isolation of CDNA clones encoding putative odorant binding proteins from the antennae of the malaria transmitting mosquito Anopheles gambiae. Insect Mol Biol. 2002:11:123-32.

31. Biessmann H, Andronopoulou E, Biessmann MR, Douris V, Dimitratos SD, Eliopoulos E, et al. The Anopheles gambiae odorant binding protein 1 (AgamOBP1) mediates indole recognition in the antennae of female mosquitoes. PLoS ONE. 2010;5:e9471.

32. Justice RW, Dimitratos $S$, Walter MF, Woods DF, Biessmann H. Sexual dimorphic expression of putative antennal carrier protein genes in the malaria vector Anopheles gambiae. Insect Mol Biol. 2003;12:581-94.

33. Li ZX, Pickett JA, Field LM, Zhou JJ. Identification and expression of odorantbinding proteins of the malaria-carrying mosquitoes Anopheles gambiae and Anopheles arabiensis. Arch Insect Biochem Physiol. 2005;58:175-89.

34. Sengul MS, Tu Z. Identification and characterization of odorant-binding protein 1 gene from the Asian malaria mosquito Anopheles stephensi. Insect Mol Biol. 2010;19:49-60.

35. Rund SS, Bonar NA, Champion MM, Ghazi JP, Houk CM, Leming MT, et al. Daily rhythms in antennal protein and olfactory sensitivity in the malaria mosquito Anopheles gambiae. Sci Rep. 2013;29(3):2494.

36. Rusconi B, Maranhao AC, Fuhrer JP, Krotee P, Choi SH, Grun F, et al. Mapping the Anopheles gambiae Odorant Binding Protein 1 (AgamOBP1) using modeling techniques, site directed mutagenesis, circular dichroism and ligand binding assays. Biochim Biophys Acta. 2012;1824:947-53.

37. Tsitsanou KE, Thireou T, Drakou CE, Koussis K, Keramioti MV, Leonidas DD, et al. Anopheles gambiae odorant binding protein crystal complex with the synthetic repellent DEET: implications for structure-based design of novel mosquito repellents. Cell Mol Life Sci. 2012;69:283-97.

38. Murphy EJ, Booth JC, Davrazou F, Port AM, Jones DN. Interactions of Anopheles gambiae odorant-binding proteins with a human-derived repellent: implication for the mode of action of $\mathrm{N}, \mathrm{N}$-diethyl-3-methylbenzamide (DEET). J Biol Chem. 2013;288:4475-85.

39. Gholizadeh S, Firooziyan S, Ladonni H, Hajiipirloo HM, Djadid ND, Hosseini A, et al. The Anopheles stephensi odorant binding protein 1 (AsteObp1) gene: a new molecular marker for biological forms diagnosis. Acta Trop. 2015;146:101-13.

40. Firooziyan S, Djadid ND, Gholizadeh S. Speculation on the possibility for introducing Anopheles stephensi as a species complex: preliminary evidence based on odorant binding protein 1 intron I sequence. Malar J. 2018;17:366.

41. Hall TA. Bioedit: a user-friendly biological sequence alignment editor and analysis program for Windows 95/98/NT. Nucleic Acids Symp Ser. 1999;41:95-8.

42. Tamura K, Stecher G, Peterson D, Filipski A, Kumar S. MEGA6: molecular evolutionary genetics analysis version 6.0. Mol Biol Evol. 2013;16:2725-9.

43. Robbins PJ, Cherniack MG. Review of the bio-distribution and toxicity of the insect repellent N, N-diethyl-m-toluamide (DEET). J Toxicol Environ Health. 1986;18:503-25.

44. Corbel V, Stankiewicz M, Pennetier C, Fournier D, Stojan J, Girard E, et al. Evidence for inhibition of cholinesterase in insect and mammalian nervous systems by the insect repellent DEET. BMC Biol. 2009;7:47.

45. Rapoport TA. Protein translocation across the eukaryotic endoplasmic reticulum and bacterial plasma membranes. Nature. 2007:450:663-9.

46. Rabouille C. Pathways of unconventional protein secretion. Trends Cell Biol. 2017:27:230-40.

\section{Publisher's Note}

Springer Nature remains neutral with regard to jurisdictional claims in published maps and institutional affiliations. 